\title{
Erratum to: Use of Modeling and Simulation Tools for Understanding the Impact of Formulation on the Absorption of a Low Solubility Compound: Ciprofloxacin
}

\author{
Marilyn Martinez, ${ }^{1,6}$ Bipin Mistry, ${ }^{1}$ Viera Lukacova, ${ }^{2}$ James E. Polli, ${ }^{3}$ Stephen W. Hoag, ${ }^{3}$ Thomas Dowling, ${ }^{4}$ \\ Ravikanth Kona, ${ }^{5}$ and Raafat Fahmy ${ }^{1}$
}

\section{Erratum to: AAPS J}

DOI 10.1208/s12248-016-9913-2

Errors were inadvertently made in the affiliation of several authors. These have been corrected in this erratum.

The online version of the original article can be found at http:// dx.doi.org/10.1208/s12248-016-9913-2.

\footnotetext{
${ }^{1}$ The Food and Drug Administration, Rockville, MD 20855, USA.

${ }^{2}$ Simulations Plus, Inc., 42505 10th Street West, Lancaster, CA 93534, USA.

${ }^{3}$ Department of Pharmaceutical Sciences, University of Maryland Baltimore, Baltimore, MD 21201, USA.

${ }^{4}$ Department of Pharmacy Practice, Ferris State University, Big Rapids, MI 49307, USA.

${ }^{5}$ Division of Formulation Development, Actavis Inc., Parsippany, NJ 07054, USA.

${ }^{6}$ To whom correspondence should be addressed. (e-mail: marilyn.martinez@fda.hhs.gov)
} 